\title{
Treadmill exercise with bone marrow stromal cells transplantation facilitates neuroprotective effect through BDNF-ERK1/2 pathway in spinal cord injury rats
}

\author{
You-Mi Kim', Jun-Jang Jin², Sam-Jun Lee ${ }^{3}$, Tae-Beom Se0 ${ }^{4}$, Eun-Sang Ji ${ }^{2 * *}$ \\ ${ }^{1}$ Sports Science Research Institution, Korea National Sport University, Seoul, Korea \\ 2Department of Physiology, College of Medicine, Kyung Hee University, Seoul, Korea \\ ${ }^{3}$ Department of Physical Education, College of Health, Welfare, and Education, Tong Myong University, Busan, Korea \\ ${ }^{4}$ Department of Kinesiology, College of Natural Science, Jeju National University, Jeju, Korea
}

Transplantation of bone marrow stromal cells (BMSCs) has been known as one of the effective therapeutic methods for functional recovery of spinal cord injury (SCI). Treadmill exercise also facilitates the functional recovery of SCI. Previously, we reported that combination of BMSCs transplantation with treadmill exercise potentiated the locomotor function in SCI rats. In the present study, we investigated whether recovery effect of BMSCs transplantation or treadmill exercise appears through the brain-derived neurotrophic factor (BDNF)-extracellular signal-regulated kinases 1/2 (ERK1/2) pathway. The spinal cord contusion injury was performed at the T9-T10 level using the impactor. Cultured BMSCs were transplanted directly into the lesion 1 week after SCI. Treadmill exercise was performed 6 days per a week for 6 weeks. Western blot for Bax, Bcl-2, BDNF, tyrosine kinase B (TrkB), and phos- phorylated ERK1/2 (p-ERK1/2), phosphorylated JNK was performed. In the present results, combination of BMSCs transplantation with treadmill exercise potently decreased Bax expression, potently increased $\mathrm{Bcl}-2$ expression, and potently enhanced BDNF and TrkB expressions in the injured spinal cord. Combination of BMSCs transplantation with treadmill exercise further facilitated $\mathrm{p}$-ERK1/2 and $\mathrm{p}$-c-Jun expression levels. The present findings demonstrated the synergistic effect of treadmill exercise on neuroregenerative effect of BMSCs transplantation appeared through the activation of BDNF-ERK1/2 pathway in SCI.

Keywords: Spinal cord injury, Treadmill exercise, Bone marrow stromal cells, Brain-derived neurotrophic factor, Extracellular signal-regulated kinases $1 / 2$

\section{INTRODUCTION}

Spinal cord injury (SCI) is a permanent trauma causing general paralysis and paraplegia due to the cavity formation, cell death, demyelination and inflammation around the injured area (Jung et al., 2016). Many researchers have been trying to find out new therapeutic method for regeneration of the injured spinal cord, and various kinds of cells were tried for transplantation (Tetzlaff et al., 2011). Of these, bone marrow stromal cells (BMSCs) have been regarded as one of the most effective candidates for the improvement of neurological function after SCI. Nakano et al. (2013) reported that BMSCs transplantation through the cerebrospinal fluid after the subacute and chronic SCI effectively promoted survival and neurite outgrowth of cultured neurons as well as axonal regeneration within the cavity. BMSCs grafting within the cavity after SCI induced axonal sprouting in the cavity by increase of brain-derived neurotrophic factor (BDNF) expression level (Kim et al., 2017; Ritfeld et al., 2015).

BDNF, a member of neurotrophin family of growth factors, binds to tyrosine kinase $\mathrm{B}(\operatorname{TrkB})$ and then mediates the diverse effects. Interaction of BDNF with TrkB signaling pathway modulates cell survival, axonal sprouting, and functional recovery (Shin et al., 2016; Tu et al., 2018). Wu et al. (2016) showed that increase of BDNF/TrkB expression regulated neuroplasticity and
${ }^{*}$ Corresponding author: Eun-Sang Ji iD https://orcid.org/0000-0001-6135-7183 Department of Physiology, College of Medicine, Kyung Hee University, 26 Kyungheedae-ro, Dongdaemun-gu, Seoul 02447, Korea Tel: +82-2-961-0282, Fax: +82-2-964-2195, E-mail: wldmstkd11@hanmail.net Received: April 2, 2018 / Accepted: May 17, 2018
This is an Open Access article distributed under the terms of the Creative Commons Attribution Non-Commercial License (http://creativecommons.org/licenses/by-nc/4.0/) which permits unrestricted non-commercial use, distribution, and reproduction in any medium, provided the original work is properly cited. 
motor function recovery after SCI.

Physical activities, such as voluntary wheel running, swimming, weight bearing, and treadmill exercise improve activity of neuronal and non-neuronal cell and axonal regrowth after peripheral and central nerve injury (Cotman et al., 2007; Liao et al., 2017; Wheaton et al., 2013). Previous studies reported that exercise might be a critical mediator to improve functional recovery and pain-related cellular reactions with the cell survival and differentiation in the injured area (Fouad et al., 2000; Sumizono et al., 2018). Increase of cell survival rate in the injured spinal cord is closely associated with secretion of neurotrophic factors (Yang et al., 2016).

Treadmill exercise facilitated recovery of motor function by increasing BDNF and phosphorylated extracellular signal-regulated kinases 1/2 (p-ERK1/2) levels after SCI (Jung et al., 2014; Oh et al., 2009). ERK1/2 belongs to mitogen-activated protein kinase family, which consists of two downstream molecules, such as JNK and c-Jun, which regulate cell survival and neuroprotective function in the nervous system. In the present study, we investigated whether combined treadmill exercise with BMSCs transplantation activates ERK1/2 downstream molecules related with neuroprotective function after SCI.

\section{MATERIALS AND METHODS}

\section{Animals and treatments}

Forty adult male Sprague-Dawley rats (180 $\pm 10 \mathrm{~g}, 6$ weeks old) were used. The experimental procedures were performed in accordance with the animal care guidelines of the National Institute of Health and the Korean Academy of Medical Sciences. The animals were housed under controlled temperature $\left(23^{\circ} \mathrm{C} \pm 2^{\circ} \mathrm{C}\right)$ and lighting (8:00 a.m. to 8:00 p.m.) conditions with food and water available ad libitum. The animals were randomly divided into four groups ( $\mathrm{n}=10$ in each group): SCI group, SCI and treadmill exercise group, SCI and BMSCs transplantation group, and SCI and BMSCs transplantation with treadmill exercise group.

\section{Spinal cord contusion injury}

Spinal cord contusion injury was induced according to the previously described method (Kim et al., 2017). The rats were anesthetized with Zoletil $50(10 \mathrm{mg} / \mathrm{kg}$, intraperitoneally; Vibac Laboratories, Carros, France). A laminectomy was performed to expose the spinal cord at thoracic level T9-10 without disrupting the dura. A contusion injury was created using the New York University Impactor System (NYU impactor, New York, NY,
USA) by dropping a 10-g impactor from 2.5 -cm height onto the exposed dura.

\section{BMSCs culture and transplantation}

BMSCs were obtained by perfusion through femur and tibia of 4-week-old rats, as the previously described method (Shin et al., 2016). Cells were cultured in Dulbecco's modified Eagle's medium with $20 \%$ fetal calf serum. Seven days later, cultured BMSC at a density of $5 \times 10^{6}$ in $50 \mu \mathrm{L}$ phosphate-buffered saline (PBS) was injected into the cavity of the injured spinal cord using a micro syringe with a 30-gauge needle. For the control, PBS without BMSCs was injected as the same manner.

\section{Treadmill exercise protocol}

The rats in the exercise groups were made to run at a speed of 8 $\mathrm{m} / \mathrm{min}$ on a horizontal platform for 30 min once a day, 6 days a week for 6 weeks.

\section{Western blot analysis}

The animals were fully anesthetized using Zoletil $50(10 \mathrm{mg} /$ $\mathrm{kg}$, intraperitoneally; Vibac Laboratories), and then the spinal cord at T9-10 level was dissected. Western blot analysis was performed according to the previously described method (Jung et al., 2016). The spinal cord tissues covering approximately $1 \mathrm{~cm}$ of the rostral and caudal spinal cord at the injury area were prepared and washed with ice-cold PBS and sonicated in 400-600 mL of Triton lysis buffer. Protein separation was performed using a $10 \%$ polyacrylamide with $0.05 \%$ bis-acrylamide. Proteins were then transferred to nitrocellulose and the blots were probed with anti-Bax rabbit polyclonal antibody (1:1,500, Santa Cruz Biotechnology, Santa Cruz, CA, USA), anti-Bcl-2 mouse monoclonal antibody (1:1,500, Santa Cruz Biotechnology), anti-BDNF rabbit polyclonal antibody (1:1,000, Santa Cruz Biotechnology), anti-TrkB rabbit polyclonal antibody (1:1,000, Santa Cruz Biotechnology), anti-p-ERK1/2 rabbit monoclonal antibody (1:3,000, Cell Signaling Technology, Beverly, MA, USA), anti-p-JNK rabbit monoclonal antibody (1:2,000, Cell Signaling Technology), anti-p-c-Jun rabbit monoclonal antibody (1:2,000, Cell Signaling Technology), and anti- $\beta$-actin mouse monoclonal antibody $(1: 3,000$, Santa Cruz Biotechnology). Peroxidase anti-rabbit IgG (1:5,000 Vector Laboratories, Burlingame, CA, USA), and peroxidase anti-mouse $\operatorname{IgG}(1: 5,000$, Vector Laboratories) were used as the secondary antibodies. 


\section{Data analysis}

The detected bands were calculated densitometrically using Molecular Analyst, version 1.4.1 (Bio-Rad, Hercules, CA, USA). Statistical analysis was performed using one-way analysis of variance followed by Duncan post hoc test. The results were expressed as the mean \pm standard error of the mean. Significance was set at $P<0.05$.

\section{RESULTS}

\section{Bax and $\mathrm{BCl}-2$ expressions}

The levels of Bax and Bcl-2 in the injured spinal cord are presented in Fig. 1. Bax level was decreased by treadmill exercise or BMSCs transplantation. Combination of treadmill exercise with BMSCs showed more potent decrement on Bax expression. Bcl-2 level was enhanced by treadmill exercise or BMSCs transplantation. Combination of treadmill exercise with BMSCs showed more potent enhancement on $\mathrm{Bcl}-2$ expression.

\section{BDNF and TrkB expressions}

The levels of BDNF and TrkB in the injured spinal cord are presented in Fig. 2. BDNF and TrkB levels were increased by treadmill exercise or BMSCs transplantation. Combination of treadmill exercise with BMSCs showed more potent increment on
BDNF and TrkB expressions.

\section{p-ERK1/2, p-JNK, and p-C-Jun expressions}

The levels of p-ERK1/2, p-JNK, and p-c-Jun in the injured spinal cord are presented in Fig. 3. p-ERK1/2 and p-c-Jun levels were increased by treadmill exercise or BMSCs transplantation. Combination of treadmill exercise with BMSCs showed more potent increment on p-ERK1/2 and p-c-Jun expressions. p-JNK level was decreased by treadmill exercise or BMSCs transplantation. Combination of treadmill exercise with BMSCs showed more potent decrement on p-JNK expression.

\section{DISCUSSION}

Repair of damaged tissue, axonal elongation, inhibiting of cell death occur by BMSCs transplantation (Ide et al., 2010) or voluntary exercise (Fouad et al., 2000) after SCI. In the present study, combination of BMSCs transplantation with treadmill exercise potently decreased Bax expression and potently increased Bcl-2 expression in the injured spinal cord. Excessive apoptosis causes a variety of neurodegenerative conditions in the injured spinal cord (Hwang et al., 2013; Yong et al., 1998). Apoptosis is a form of programmed cell death and also induced in various states of human diseases. Apoptosis is identified by activation of the cysteine
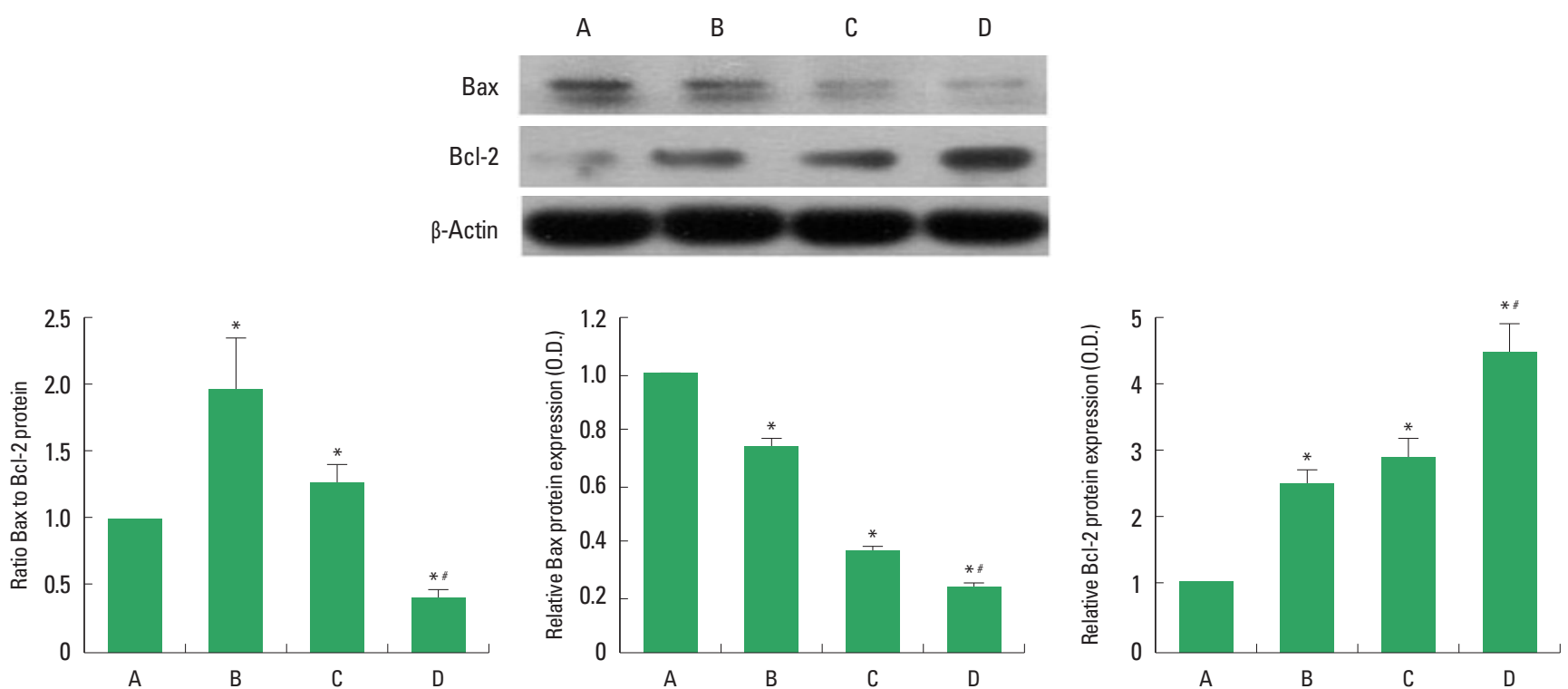

Fig. 1. Bax and Bcl-2 levels in the spinal cord injury (SCI). Upper left panel: Representative expressions of Bax, Bcl-2, and $\beta$-actin. Lower panel: Relative Bax and Bcl-2 expressions. A, SCl group; B, SCl and treadmill exercise group; C, SCl and bone marrow stromal cells (BMSCs) transplantation group; D, SCI and BMSCs transplantation with treadmill exercise group. Values are presented as mean \pm standard error of the mean. ${ }^{*} P<0.05$ compared to the SCl group. ${ }^{\#} P<0.05$ compared to the SCl and BMSCs transplantation group. 

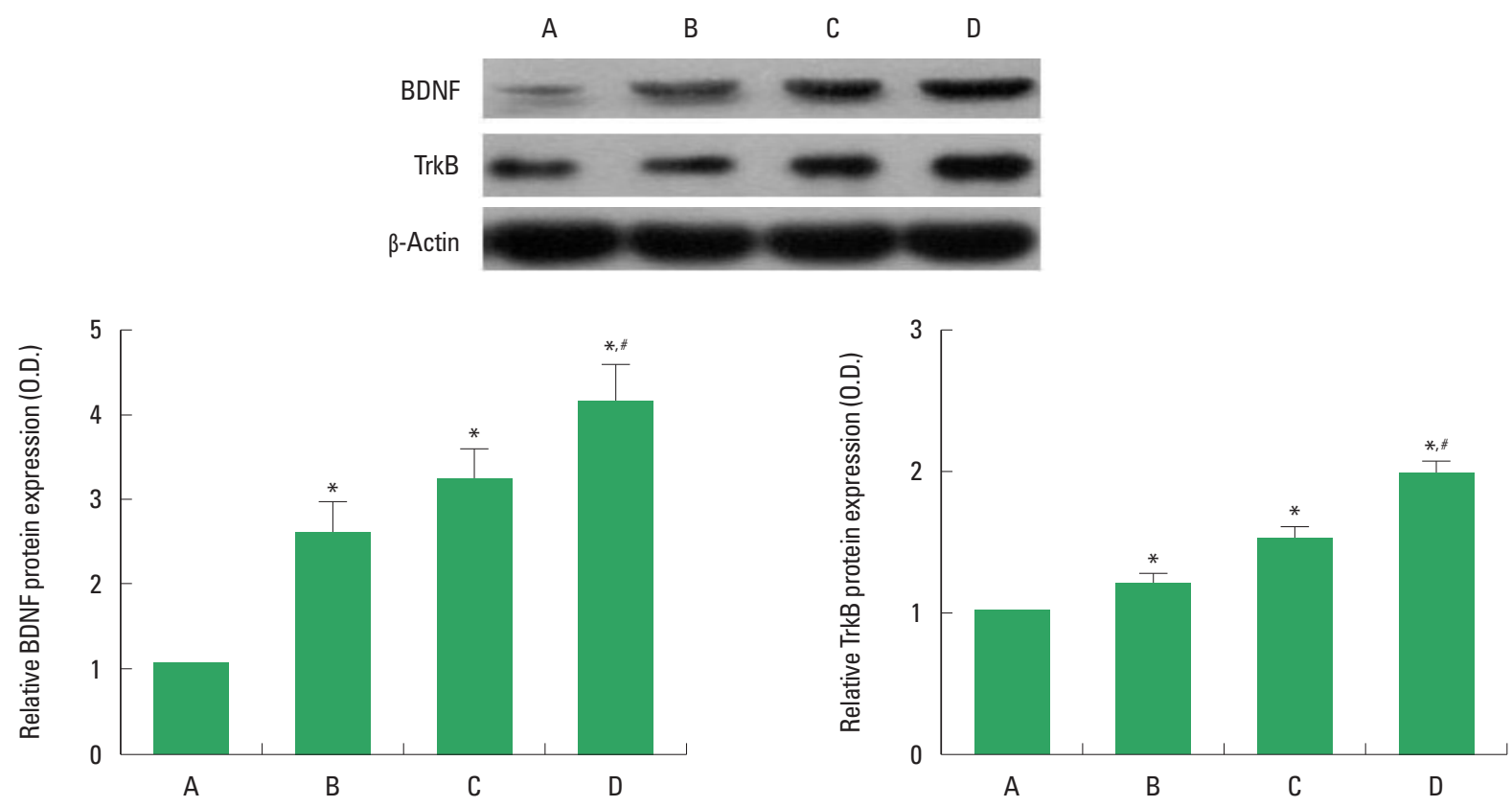

Fig. 2. Brain-derived neurotrophic factor (BDNF) and tyrosine kinase B (TrkB) levels in the spinal cord injury (SCI). Upper panel: Representative expression of BDNF, TrkB2, and $\beta$-actin. Lower panel: Relative expressions of BDNF and TrKB. A, SCl group; B, SCl and treadmill exercise group; C, SCl and bone marrow stromal cells (BMSCs) transplantation group; D, SCl and BMSCs transplantation with treadmill exercise group. Values are presented as mean \pm standard error of the mean. ${ }^{*} P<0.05$ compared to the SCl group. ${ }^{*} P<0.05$ compared to the SCl and BMSCs transplantation group.
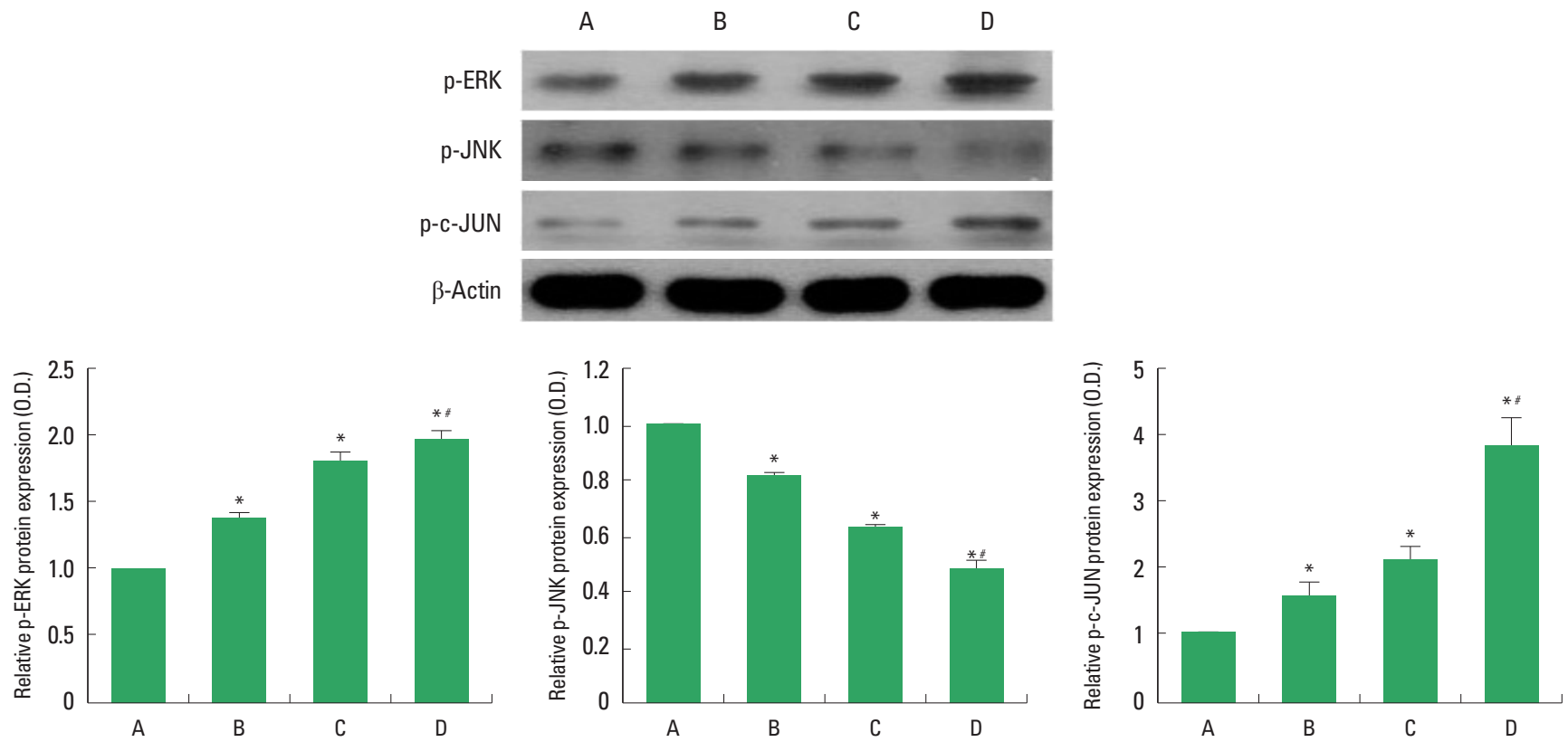

Fig. 3. Phosphorylated extracellular signal-regulated kinases 1/2 (p-ERK1/2), p-JNK and p-c-Jun levels in the spinal cord injury (SCI). Upper panel: Representative expressions of p-ERK1/2, p-JNK, p-c-Jun, and $\beta$-actin. Lower panel: Relative p-ERK1/2, p-JNK and p-c-Jun expressions. A, SCI group; B, SCI and treadmill exercise group; C, SCl and bone marrow stromal cells (BMSCs) transplantation group; D, SCl and BMSCs transplantation with treadmill exercise group. Values are presented as mean \pm standard error of the mean. ${ }^{*} P<0.05$ compared to the SCI group. ${ }^{*} P<0.05$ compared to the $S C l$ and $\mathrm{BMSC}$ s transplantation group. 
protease family such as Bax and Bcl-2 (Emery et al., 1998). It has been well known that $\mathrm{Bcl}-2$, antiapoptotic protein, inhibits the process of apoptotic cell death (Kuwana and Newmeyer, 2003), meanwhile Bax, pro-apoptotic protein, leads to acceleration of apoptotic cell death (Shin et al., 2016).

In order to examine the neuroprotective effect of combination of BMSCs transplantation with treadmill exercise after SCI, we measured the expression levels of BDNF and TrkB. In the present study, combination of BMSCs transplantation with treadmill exercise potently enhanced BDNF and $\operatorname{TrkB}$ expressions in the injured spinal cord. Neurotrophic factors such as BDNF, nerve growth factor, and neurotrophin-3 are critical molecules for neuroprotection. Transplanted BMSCs after SCI enhanced survival of spinal motor neurons about $64 \%$ (Ritfeld et al., 2015). Increased BDNF by exercise improved functional recovery in patient with incomplete SCI (Leech and Hornby, 2017). Present results suggest that BMSCs transplantation with physical exercise might be a new therapeutic strategy for function recovery from SCI.

BDNF-TrkB signaling pathway is the upstream molecule of ERK1/2 in the nervous system. ERK1/2 is related with neuronal survival, differentiation, and neurite outgrowth (Oh et al., 2009), and its downstream molecule is composed of two proteins with opposing functions: one is JNK protein involved in apoptosis and axonal retraction and the other is c-Jun protein related to cell survival and neuroplasticity. ERK1/2 activation in the injured spinal cord regulated inflammatory pain in sensory neurons of the spinal cord (Xu et al., 2008). Blockade of ERK1/2 decreased sprouting of axons in corticospinal tract around lesioned area (Oh et al., 2009). In the present study, transplantation of BMSCs or treadmill exercise increased p-ERK1/2 and p-c-Jun levels in the injured spinal cord. Combination of BMSCs transplantation with treadmill exercise further facilitated p-ERK1/2 and p-c-Jun levels. Treadmill exercise is known to increase p-ERK1/2 level in the spinal cord and the motor cortex 1 and 2 weeks after SCI, and the increment of p-ERK1/2 extends corticospinal tract fibers around the lesioned area (Oh et al., 2009). Treatment with BMSCs combined with plumbagin improved locomotor function and enhanced Nrf2, p-Akt, and p-ERK1/2 expressions in the SCI area (Yang et al., 2016). We speculate that activation of ERK1/2 pathway is implicated in the regeneration of the injured spinal cord.

Previously, we reported that treadmill exercise potentiated the improving effect of BMSCs transplantation on locomotor function in the SCI rats, and this potentiating effect of treadmill exercise might be ascribed to the enhancement of BDNF expression in the SCI rats (Kim et al., 2017). In the present results, both BMSCs and treadmill exercise activated ERK1/2 pathway and treadmill exercise showed the enhancing effect of BMSCs-induced activation of ERK1/2 pathway. The present findings demonstrated the synergistic effect of treadmill exercise on neuroregenerative effect of BMSCs transplantation appeared through the activation of BDNF-ERK1/2 pathway in SCI.

\section{CONFLICT OF INTEREST}

No potential conflict of interest relevant to this article was reported.

\section{ACKNOWLEDGMENTS}

This work was supported by the National Research Foundation of Korea Grant funded by the Korean Government (NRF2014S1A5B5A02013292).

\section{REFERENCES}

Cotman CW, Berchtold NC, Christie LA. Exercise builds brain health: key roles of growth factor cascades and inflammation. Trends Neurosci 2007;30:464-472.

Emery E, Aldana P, Bunge MB, Puckett W, Srinivasan A, Keane RW, Bethea J, Levi AD. Apoptosis after traumatic human spinal cord injury. J Neurosurg 1998;89:911-920.

Fouad K, Metz GA, Merkler D, Dietz V, Schwab ME. Treadmill training in incomplete spinal cord injured rats. Behav Brain Res 2000;115:107113.

Hwang L, Choi IY, Kim SE, Ko IG, Shin MS, Kim CJ, Kim SH, Jin JJ, Chung JY, Yi JW. Dexmedetomidine ameliorates intracerebral hemorrhageinduced memory impairment by inhibiting apoptosis and enhancing brain-derived neurotrophic factor expression in the rat hippocampus. Int J Mol Med 2013;31:1047-1056.

Ide C, Nakai Y, Nakano N, Seo TB, Yamada Y, Endo K, Noda T, Saito F, Suzuki Y, Fukushima M, Nakatani T. Bone marrow stromal cell transplantation for treatment of sub-acute spinal cord injury in the rat. Brain Res 2010;1332:32-47.

Jung SY, Kim DY, Yune TY, Shin DH, Baek SB, Kim CJ. Treadmill exercise reduces spinal cord injury-induced apoptosis by activating the PI3K/ Akt pathway in rats. Exp Ther Med 2014;7:587-593.

Jung SY, Seo TB, Kim DY. Treadmill exercise facilitates recovery of locomotor function through axonal regeneration following spinal cord injury in rats. J Exerc Rehabil 2016;12:284-292.

Kim YM, Seo TB, Kim CJ, Ji ES. Treadmill exercise with bone marrow 
stromal cells transplantation potentiates recovery of locomotor function after spinal cord injury in rats. J Exerc Rehabil 2017;13:273-278.

Kuwana T, Newmeyer DD. Bcl-2-family proteins and the role of mitochondria in apoptosis. Curr Opin Cell Biol 2003;15:691-699.

Leech KA, Hornby TG. High-intensity locomotor exercise increases brainderived neurotrophic factor in individuals with incomplete spinal cord injury. J Neurotrauma 2017;34:1240-1248.

Liao CF, Yang TY, Chen YH, Yao CH, Way TD, Chen YS. Effects of swimming exercise on nerve regeneration in a rat sciatic nerve transection model. Biomedicine (Taipei) 2017;7:3.

Nakano N, Nakai Y, Seo TB, Homma T, Yamada Y, Ohta M, Suzuki Y, Nakatani T, Fukushima M, Hayashibe M, Ide C. Effects of bone marrow stromal cell transplantation through CSF on the subacute and chronic spinal cord injury in rats. PLoS One 2013;8:e73494.

Oh MJ, Seo TB, Kwon KB, Yoon SJ, Elzi DJ, Kim BG, Namgung U. Axonal outgrowth and Erk1/2 activation by training after spinal cord injury in rats. J Neurotrauma 2009;26:2071-2082.

Ritfeld GJ, Patel A, Chou A, Novosat TL, Castillo DG, Roos RA, Oudega $M$. The role of brain-derived neurotrophic factor in bone marrow stromal cell-mediated spinal cord repair. Cell Transplant 2015;24:2209-2220.

Shin MS, Park HK, Kim TW, Ji ES, Lee JM, Choi HS, Kim MY, Kim YP. Neuroprotective effects of bone marrow stromal cell transplantation in combination with treadmill exercise following traumatic brain injury. Int Neurourol J 2016;20(Suppl 1):S49-56.

Sumizono M, Sakakima H, Otsuka S, Terashi T, Nakanishi K, Ueda K, Takada S, Kikuchi K. The effect of exercise frequency on neuropathic pain and pain-related cellular reactions in the spinal cord and midbrain in a rat sciatic nerve injury model. J Pain Res 2018;11:281-291.
Tetzlaff W, Okon EB, Karimi-Abdolrezaee S, Hill CE, Sparling JS, Plemel JR, Plunet WT, Tsai EC, Baptiste D, Smithson LJ, Kawaja MD, Fehlings MG, Kwon BK. A systematic review of cellular transplantation therapies for spinal cord injury. J Neurotrauma 2011;28:1611-1682.

Tu WZ, Li SS, Jiang X, Qian XR, Yang GH, Gu PP, Lu B, Jiang SH. Effect of electro-acupuncture on the BDNF-TrkB pathway in the spinal cord of CCI rats. Int J Mol Med 2018;41:3307-3315.

Wheaton BJ, Noor NM, Whish SC, Truettner JS, Dietrich WD, Zhang M, Crack PJ, Dziegielewska KM, Saunders NR. Weight-bearing locomotion in the developing opossum, Monodelphis domestica following spinal transection: remodeling of neuronal circuits caudal to lesion. PLoS One 2013;8:e71181.

Wu Q, Cao Y, Dong C, Wang H, Wang Q, Tong W, Li X, Shan C, Wang T. Neuromuscular interaction is required for neurotrophins-mediated locomotor recovery following treadmill training in rat spinal cord injury. PeerJ 2016;4:e2025.

Xu Q, Garraway SM, Weyerbacher AR, Shin SJ, Inturrisi CE. Activation of the neuronal extracellular signal-regulated kinase 2 in the spinal cord dorsal horn is required for complete Freund's adjuvant-induced pain hypersensitivity. J Neurosci 2008;28:14087-14096.

Yang W, Yang Y, Yang JY, Liang M, Song J. Treatment with bone marrow mesenchymal stem cells combined with plumbagin alleviates spinal cord injury by affecting oxidative stress, inflammation, apoptotis and the activation of the Nrf2 pathway. Int J Mol Med 2016;37:1075-1082.

Yong C, Arnold PM, Zoubine MN, Citron BA, Watanabe I, Berman NE, Festoff BW. Apoptosis in cellular compartments of rat spinal cord after severe contusion injury. J Neurotrauma 1998;15:459-472. 